\title{
Molecular Epidemiologic Factors Contributing to Guinolone Resistance in Clinical Multidrug-resistant Klebsiella Pneumoniae Isolates from Shanghai, China
}

\author{
Yan Wang ( $\nabla$ wangyan_hf@126.com ) \\ Fudan University https://orcid.org/0000-0002-8457-5287 \\ Guoping Cai \\ Fudan University Jinshan Hospital \\ Jinan Zhang \\ Shanghai Jiankang Medical University \\ Xiaogang Xu \\ Fudan University Huashan Hospital Institute of Antibiotics \\ Hongzhou Lu
}

Fudan University Affiliated Public Health Clinical Center: Shanghai Public Health Clinical Center

\section{Research}

Keywords: Molecular Epidemiologic Factors, Guinolone Resistance, Clinical Multidrug-resistant, Klebsiella pneumoniae

Posted Date: May 6th, 2021

DOl: https://doi.org/10.21203/rs.3.rs-492410/v1

License: (c) (i) This work is licensed under a Creative Commons Attribution 4.0 International License.

Read Full License 
Molecular epidemiologic factors contributing to quinolone resistance in clinical multidrug-resistant Klebsiella pneumoniae isolates from Shanghai, China Yan Wang ${ }^{1+*}$, Guoping $\mathrm{Cai}^{2^{+}}$, Jinan Zhang ${ }^{3}$, Xiaogang $\mathrm{Xu}^{4}$, Hongzhou $\mathrm{Lu}^{5^{*}}$

${ }^{\dagger}$ Yan Wang and Guoping Cai contributed equally to this work.

*Co-corresponding authors:

Research Center for Clinical Medicine, Jinshan Hospital, Fudan University, 1508 Longhang Road, Jinshan District, Shanghai 201508, China. wangyan hf@126.com (YW).

Department of infections and immunity, Shanghai Public Health Clinical Center, Fudan University, 2901 Caolang Road, Jinshan District, Shanghai 201508, China. luhongzhou@fudan.edu.cn (H-ZL).

Full list of author information is available at the end of the article. 


\section{Abstract}

2 Background: The soaring quinolone-resistance rate of Klebsiella pneumoniae, a common pathogen in

3 immunocompromised individuals, has seriously undermined the wide applications of antimicrobials of

4 this class. This study aimed to investigate the emerging key contributors to quinolone resistance in

5 multidrug resistant $K$. pneumoniae (MDR-KP) isolates from a clinical setting with continuing

6 point-source infection outbreaks.

7 Methods: A total of $34 \mathrm{~K}$. pneumoniae isolates, including 30 carbapenem-resistant $K$. pneumoniae

8 (CRKP) isolates were randomly selected from a teaching hospital participating in an ongoing Bacterial

9 Resistance Surveillance Project in Shanghai, China. Investigations included antimicrobial susceptibility tests, MLST and wzi-genotyping, PCR and DNA sequencing for identification of resistance determinants, sequence analyses using MEGA7.0 software, and clinical information retrieving through electronic medical records.

Results: Two predominant high-risk resistant clones, ST11-wzi64 and ST15-wzi19/wzi24, from 30 CRKP isolates caused three point-source nosocomial outbreaks in intensive care unit and neurosurgery department potentially by respiratory-route, promoting the co-selection and evolution of multidrug resistant determinants. Multiple quinolone resistance-determining region (QRDR) mutations occurred in isolates of ST15 (S83F, D87A; S80I), ST11 (S83I, D87G; S80I), and ST218 (D87A; S80I). Plasmid-mediated quinolone resistance determinants, qnrS1, $a a c\left(6^{\prime}\right)-I b-c r$, oqxAB, were detected in 32 (94.1\%) isolates alone or in combination, accompanied with $\beta$-lactamases, 16S rRNA methylases, and putrescine $A B C$ permeases. $A c r R, A c r A B$ transcriptional repressor, was insertion-inactivated by IS5-transposase in isolates of ST11. Thirteen ompK36 variants associated with specific ST $(n=7)$ and wzi-allele $(n=9)$ clustered into 10 (sub)lineages in the phylogenetic tree possibly affecting the MDR phenotype and infection outcome of isolates. Isolates of ST11, ST15, and ST218 had frameshift disruptions in OmpK35 coupled with specific GD-insertion at position 134-135 in OmpK36, all showing distinct microevolution clusters of ompK36 genotypes. Seven quinolone-susceptible isolates kept the 

NDM-1-coproducers) and ST65-wzi72.

28 Conclusions: Under multiple selective pressures, accumulation of mutations of three types (QRDR, 29 AcrR, OmpK36/OmpK35) and acquisition of resistance-conferring genes contribute to quinolone-resistance in clinical MDR-KP isolates, reinforcing the importance of continued epidemiologic surveillance on the evolution and transmission of these isolates. Our findings provided detailed mechanistic analyses and epidemiologic implications for further infection control and antibiotic stewardship initiatives.

\section{Introduction}

35 Quinolone antimicrobials are a class of synthetic broad-spectrum agents applicable to a range of gram-negative bacilli (GNB) infections in humans or animals. Klebsiella pneumoniae, an important zoonotic pathogen, is naturally susceptible to these agents; however, resistance emerged worldwide with use, which was primarily due to the successive quinolone resistance-determining region (QRDR) mutations on chromosomes ${ }^{[1]}$ and secondly, facilitated by the wide dissemination of plasmid-mediated quinolone resistance (PMQR) determinants ${ }^{[2]}$. Furthermore, chromosomal mutations may also cause decreased outer-membrane permeability ${ }^{[3,4]}$ and PMQR-encoding plasmids often co-transfer additional resistance determinants, both of which could confer cross-resistance phenotype for antimicrobial agents of multiple families. Thus, $K$. pneumoniae is potentially so important a reservoir for multidrug resistance (MDR) determinants that clinicians were increasingly challenged for effectively treating life-threatening infections caused by those, mainly carbapenem-resistant $K$. pneumoniae (CRKP) isolates that places a significant burden on patient health and healthcare systems ${ }^{[5,6]}$, particularly in low-middle income countries $^{[5]}$, a daily issue for the management of antimicrobial therapy for critically ill patients. 
51 for the rapid and accurate diagnosis of epidemic high-risk clones to improve clinical outcome and cut

52 healthcare costs. Data from China Antimicrobial Surveillance Network (CHINET) revealed that $K$.

53 pneumoniae predominated over major GNB increasingly over years, rising to the first-place pathogen

54 in respiratory tract specimens since $2017^{[7]}$. Fluoroquinolones, among the most extensively used

55 antimicrobials in China, have limited application in some clinical settings as the increasing resistance

56 over time, e.g. the ciprofloxacin-resistance rates of $K$. pneumoniae from respiratory tract (31.1\%),

57 urine (46.5\%), blood (45.9\%) and cerebrospinal fluid (59.4\%) in $2020^{[7]}$. Furthermore, the aggregated

58 resistance rates to fluoroquinolone in $K$. pneumoniae invasive infections in 2017 worldwide varied

59 geographically, ranging from low- (4\%, Australia; $10 \%$, USA) to high- $(29 \%$, France; $42 \%$, China) or even

60 extremely high-rate $\left(87 \%\right.$, Russia ${ }^{[8]}$. Herein, we investigated the molecular mechanisms underlying

61 quinolone resistance in MDR K. pneumoniae (MDR-KP) isolates from a 700-bed tertiary teaching

62 hospital participating in an ongoing Bacterial Resistance Surveillance Project in Shanghai, an

63 international metropolis in China, to shed light on the new evolution trend of association between

resistance to quinolones and to other agents as well the balance between antimicrobial resistance and

competitive fitness, for epidemiologic, infection control, and antibiotic stewardship initiatives.

\section{Materials and Methods}

\section{Patients, Isolates and Antibiograms}

Nosocomial infection was defined as infection occurring $>48 \mathrm{~h}$ after admission to the hospital. A total of 34 K. pneumoniae isolates from nosocomial infections between January and March 2017 were randomly selected; pertinent information regarding the infections caused by these isolates was retrieved through electronic medical records. The routine antibiograms for all isolates were determined using a disc diffusion assay following CLSI (2017) guidelines ${ }^{[9]}$, and further confirmed using 
74 following antimicrobials were tested, including ciprofloxacin, amikacin, gentamicin, ampicillin,

75 ampicillin-sulbactam, piperacillin, piperacillin-tazobactam, cefazolin, cefaclor, cefuroxime, cefotaxime,

76 ceftazidime, cefepime, trimethoprim-sulfamethoxazole, fosfomycin, imipenem, and meropenem. An

77 isolate assigned to the intermediately susceptible, susceptible dose dependent, or resistant category

78 was defined as nonsusceptible to the drug tested in this study; and nonsusceptible to imipenem

79 and/or meropenem was regarded as CRKP.

80 Homology Analysis of K. pneumoniae

81 MLST targeting seven housekeeping genes (gapA, infB, $m d h$, pgi, phoE, rpoB, and ton $B)^{[10]}$, together

82 with wzi-genotyping for inference of the capsular $(\mathrm{K})$ types ${ }^{[11]}$, was performed to investigate the

83 epidemiological associations of the selected isolates. ST and wzi-allele were determined by querying

84 the database maintained by the Pasteur Institute.

PCR amplification was performed using the following primers (Table S1): i) QRDR (gyrA, parC) ${ }^{[12]}$ and PMQR genes (qnrA, qnrB, qnrC, qnrD, qnrS, aac $\left(6^{\prime}\right)-l b-c r, q e p A$, oqxAB $)^{[13-19]}$. ii) $\beta$-lactamase genes encoding extended-spectrum $\beta$-lactamase $(\mathrm{ESBL})\left(b / a_{\mathrm{CTX}-\mathrm{M}}, b / a_{\mathrm{SHV}}, b / a_{\mathrm{TEM}}, b / a_{\mathrm{OXA}}\right)^{[20]}$, plasmid-borne AmpC $\beta$-lactamases $\left(b / a_{\mathrm{ACC}}, b / a_{\mathrm{CIT}}, b / a_{\mathrm{DHA}}, b / a_{\mathrm{EBC}}, b / a_{\mathrm{FOX}}, b / a_{\mathrm{MOX}}\right)^{[21]}$, and carbapenemases $\left(b / a_{\mathrm{KPC}}, b / a_{\mathrm{VIM}}\right.$,

$\left.90 b / a_{\mathrm{IMP}}, b / a_{\mathrm{NDM}}, b / a_{\mathrm{OXA}-48}\right)^{[22-23]}$. iii) $16 \mathrm{~S}$ rRNA methylase genes $\left(b / a_{\mathrm{armA}}, b / a_{\mathrm{rmtB}}\right)^{[24]}$. iv) The coding

91 sequences of outer membrane (OM) proteins, OmpK35 and OmpK36 ${ }^{[25]}$. v) The complete sequence of

$92 A c r A B$ efflux pump repressor $(A c r R)$ gene ${ }^{[26]}$. Foreign DNA segments inserted in the target genes were identified by primer walking with customized primers.

\section{DNA Sequencing and Phylogenetic Analyses}

95 PCR products were submitted for direct sequencing by 3730xI DNA Analyzer ( $A B I, U S A)$ and compared 96 with reference sequences available in the GenBank database using BLAST searches. Cloning and 97 sequencing studies were given to samples with weak or bimodal amplification. DNA sequences were analyzed using the EditSeq program in the DNASTAR software package (Madison, WI). Sequence 
alignments were performed using the CLUSTAL W algorithm and phylogenetic trees were constructed using the Neighbor-Joining method with 1,000 pseudoreplicate data sets in MEGA 7.0 software $^{[27]}$.

\section{Results}

\section{Specimens and Patients}

The specimens of sputum $(n=22,68.8 \%)$, urine, blood, and catheter drainage fluid were collected from inpatients in ICU ( $n=18)$ and neurosurgery department (ND) ( $n=14)$ with a median age of 67 and 52 years, respectively $(P<0.01)$. In ICU, the crude mortality rate was $33.3 \%$; two of the six cases died of severe pneumonia, three of ICU-acquired sepsis, and one of intracranial infection. In ND, patients with severe brain injury were mostly subjected to ventilator-associated pneumonia (VAP) after tracheotomy, but all were eventually improved or cured, and discharged. Additionally, two isolates were recovered from infants with urinary tract infection in Pediatric Ward.

\section{Phenotypic and Genotypic Resistance Patterns}

All but one isolates were various $\beta$-lactamase producers; a total of 30 isolates $(88.2 \%)$ showed CRKP phenotypes. Isolates susceptible to ciprofloxacin $(n=7,20.6 \%)$ included $4(13.3 \%)$ of CRKPs and 3 (75\%) of carbapenem-susceptible isolates $(P<0.05)$. QRDR-mutations occurred in isolates of ST15 (S83F, D87A; S80I), ST11 (S83I, D87G; S80I) and ST218 (D87A; S80I). PMQR genes, qnrS1 ( $n=6,17.6 \%)$, $\operatorname{aac}\left(6^{\prime}\right)-\operatorname{lb}-\operatorname{cr}(\mathrm{n}=10,29.4 \%)$, and $\operatorname{oqx} A B(\mathrm{n}=20,58.8 \% ; \mathrm{n}=31,91.2 \%)$, were detected in 32 isolates (94.1\%) alone or in combination, accompanied with $\beta$-lactamases (CTX-M-14/15, TEM-1, SHV-1; KPC-2, KPC-3, NDM-1; CMY-2), 16S rRNA methylases (ArmA, RmtB), and putrescine ABC transporter permease (Potl) variants (Table 1).

\section{Genetic Analysis of AcrR}

Compared to the Spainish isolate K23 (AJ318073), isolates of ST11 (JSK7343) and all non-ST11 in this study harbored the same 7-amino-acid (aa) substitutions (P161R, G164A, F172S, R173G, L195V, F197I, and K201M) at specific positions within the AcrR protein (MK341462), as seen in 
123 fluoroquinolone-resistant strains from Singapore hospitals ${ }^{[26]}$. Additionally, one silent change Q127Q

124 (CAG $\rightarrow$ CAA) was present in isolate JSK7111 (MK341463). Unexpectedly, an abnormal PCR amplicon

125 (1879bp; MK341464) was observed in all ST11 isolates but JSK7343. IS5-like element (981bp)

126 belonging to the ISKpn26 family transposase caused an insertion-inactivation mutation within the

127 AcrR structural gene (651bp) producing an incomplete acrR pseudogene (375bp) with a missing start

$128(+276 \mathrm{bp})$ and a silent change (G441A).

\section{Porin Gene Sequence Analysis}

Sequence analysis of ompK35 genes identified 5 variants that shared $100 \%$ aa sequence identity with reference strain JX310555. Disruptions in the OmpK35 coding sequence produced two allelic pseudogenes, namely, O35K7101 (G252-A253 insertion in variant 035K7106) and 035K7118 (A86 deletion in variant $035 \mathrm{~K} 7105$ ), with an early frameshift and a series of premature stop codons. Isolate JSK7111 exhibited a panel of heterogeneous ompK35 variants. The ompK35 gene was undetected in isolate JSK7109 (Table 1). in the phylogenetic tree. Except isolates of ST15 and ST218, all had a distinct ompK36 coding sequence correlated with a specific ST (wzi) and grouped into an independent phylogenetic cluster (Figure 1; Table 1). The comparison and alignment of deduced aa sequences of representative ompK36 genes worldwide revealed that multiple differences in OmpK36 peptides specific for lineages and sublineages mainly occurred in the extracellular Loops (L4-L8); the most common mutation located in the conserved internal loop, L3, which defines the size of the pore, were insertions encoding glycine and aspartic acid at aa positions 134 and 135 (ins aa 134-135 GD) in isolates of ST15, ST11, and ST218 (Figure 2). In all isolates, sequencing revealed no type of disruptions in the OmpK36 coding sequence.

Isolates of ST15 and ST218 each independently harbored a panel of ompK36 variants as a consequence of microevolution from one common index case of infection. Four ompK36 variants in 147 isolates of ST15 formed two different evolution directions in sublineage la. In ICU, three variants for previously reported isolates ST15 (wzi24-K24) ${ }^{[11]}$ differed by one to two nt displaying an evolutionary 
process from prototype, 036K7233, to pre-epidemic intermediate, O36K7340 (CTG $\rightarrow$ TTG, L9L), and then to a novel variant, O36K7106 (CTG $\rightarrow$ TTG, L9L; TCC $\rightarrow$ TTC, S7F). In contrast to ST15 (wzi24-K24), emerging isolates ST15 (wzi19-K19) mainly epidemic to ND hold different phenotypic and genotypic resistance profiles with specific ins aa134-135 GD in L3 of variant 036K7101. Isolate JSK7111 (ST218) also held a set of heterogeneous ompK36 variants that belonged to two distinct lineages (la/b and IIla). Nine isolates of ST11 sharing the same ompK36 sequence (036K7229) clustered into lineage IV with others in China, USA, etc., despite microevolution of nt sequence existing among which, the OmpK36 sequences were comparatively conserved showing five sets of identical Loop (L4-L8) insertions and deletions that were predicted to lead to 9 additional aa residues and an increase in mass of approximately $1 \mathrm{kDa}^{[28]}$, especially a replacement of 4 -aa constellation and a insertion of 7-aa string occurring in L6 (Figure 1-3; Table 1).

\section{Discussion}

K. pneumoniae has recently achieved notoriety as a common pathogen in immunocompromised individuals due to the increasing number of serious infections and the growing scarcity of effective anti-infective treatments. In the present study, so large a quinolone-resistance armamentarium was accumulated in MDR-KP isolates from nosocomial infections that multiple genetic mechanisms and epidemiologic factors had continuously contributed to the daunting issue.

High-level resistance mutations in either of the two drug target enzymes, DNA gyrase- and topoisomerase IV-encoding genes (gyrA and parC, respectively) reduced drug binding to the enzyme-DNA complex directly blocking DNA synthesis ${ }^{[1]}$; the former and the latter were the primary and the secondary target for many quinolones in clinical use for GNB, respectively. We hereby observed that resistance $(n=25 ;$ ST15, ST11, ST218) or susceptibility ( $n=6 ;$ ST65, ST13, ST107) to ciprofloxacin in ESBL- and/or KPC-producing K. pneumoniae isolates were primarily associated with whether multiple QRDR-mutations occurred or not, indicating the emergence of clinically important resistance would not have been predicted on single-mutation molecular grounds and the additional epidemiologic factors may have come into play. Recently, food, especially fresh raw chicken, was 
175 evidenced as a reservoir of MDR-KP isolates in China ${ }^{[17,29]}$. Owing to the role of hospital environment

176 in persistence and onward transmission of $\mathrm{MDR}-\mathrm{KP}^{[30]}$, resistant isolates are prone to disseminate

177 among the dense patient population causing foodborne ${ }^{[31]}$ or airborne ${ }^{[32]}$ nosocomial outbreaks.

178 Hence, $K$. pneumoniae is increasingly recognized as a foodborne pathogen. Homology analysis

179 revealed that small outbreaks of three wzi-allele-K types (wzi19-K19, wzi24-K24, and wzi64-K14.K64)

180 occurred through nosocomial transmission of two epidemic resistant clones, ST15 and ST11, which

181 had different resistance-causing aa changes, S83F-D87A and S83I-D87G, respectively, within the

182 primary target (Figure 3; Table 1). The aqueous environment, as the biggest reservoir for MDR-KP in the hospital environment ${ }^{[30]}$, might be a source of outbreaks and persistence in the endemic setting. The high frequency (68.8\%) of sputum specimens in this study suggested that spread of aerosols containing MDR-KP isolates might sustain these outbreaks as a reservoir, leading to secondary spread, hospital-acquired pneumonia (HAP), among inpatients staying in ICU and ND. Furthermore, the accumulation of QRDR-mutations in MDR-KP clinical isolates had the potential for coselection with several antimicrobial agents and produced highly resistant strains, e.g. one patient died from ICU-acquired septicopyemia caused by a ciprofloxacin-resistant $K$. pneumoniae carbapenemase (KPC)-3 variant (T23N) producing CRKP. Thus, multiresistance was potentially augmented by ongoing selection pressures of different reservoir origins resulted from broad drug use in parallel in animal and human populations coupled with spread of MDR isolates along the food chain and from human-to-human, a fact that posed the noteworthy health risks.

PMQR determinants can confer low-level resistance that facilitates the selection of mutational high-level quinolone resistance through widening the mutation selection window and elevating the mutation prevention concentration ${ }^{[33]}$, and may promote treatment failure. In this study, a high prevalence ( $\geq 10 \%$ ) of QnrS1 occurred in clinical MDR-KP isolates to greatly protect the target enzymes from quinolone action ${ }^{[2]}$. AAC(6 $\left.6^{\prime}\right)-1 \mathrm{~b}-\mathrm{cr}$, a cr-variant (W102R, D179Y) of aminoglycoside-modifying enzyme (AME), that acetylates both aminoglycosides and certain quinolones ${ }^{[16]}$, was more commonly encountered than QnrS1 and particularly associated with ESBL CTX-M-15. No mobile quinolone-pump 
resistance-nodulation-division (RND) family, firstly identified on olaquindox-resistance plasmids in $E$. coli of veterinary origin ${ }^{[34]}$, was now widely distributed in diverse clinical MDR-KP genomes from human ${ }^{[35]}$. Here, OqxAB exited in almost all isolates and was linked with ESBL CTX-M-14/15 regardless of ciprofloxacin-susceptibility. The grouping of both oqxA and $\operatorname{oqxB}$ sequences was related to specific ST, as a result of the transmission and evolution of progenitor genes from isolates of ST11 (oqxA1B1). ATP-binding cassette $(A B C)$ transporters, a ubiquitous superfamily of integral membrane proteins, are responsible for the ATP powered translocation of many substrates across membranes, also involving in a range of other processes including DNA repair and chromosome maintenance ${ }^{[36]}$. Putrescine, a product of ornithine decarboxylation, plays multifunctional roles in regulating important cellular processes by polyamine metabolic network ${ }^{[3]]}$. The putrescine $A B C$ transporter permease variant (R248L) was primarily observed in isolates of ST11 (potl-v1) and ST15 (potl-v2), suggesting their critical physiologic functions involved in the MDR phenotype. PMQR has been found on heterogeneous plasmids in clinical and environmental isolates worldwide and appears to be spreading independently of QRDR-mutations. High-level quinolone resistance could be seen, in case two or more quinolone resistance determinants were harbored by a plasmid or isolate, e.g. isolate JSK7109 (ST290; qnrS1; oqx $\mathrm{AB}$ ) as well as a fluoroquinolone-resistant $E$. coli (overexpressing the $\mathrm{Acr} A \mathrm{~B}$ and other pumps, acquiring both QnrS1 and $\left.\mathrm{OqxAB}{ }^{[38]}\right)$.

Besides the above-mentioned factors, altered OM permeability reducing cytoplasmic drug concentrations has also been shown to generate a quinolone resistance phenotype in $K$. pneumoniae. $A c r A B$ and OqxAB, two RND efflux pumps, had differential contribution to MDR and virulence ${ }^{[39]}$; in the absence of $A c r A B$, gyrase mutations fail to produce clinically relevant levels of resistance ${ }^{[40]}$. The expression of $A c r A B$ operon was down-regulated by a transcriptional repressor, $A c r R$, whose mutation was related to $A c r A B$ overexpression and fluoroquinolone resistance in $K$. pneumoniae ${ }^{[26]}$. Despite no evidence for correlation of low acrB-expression with decreased susceptibility to carbapenems ${ }^{[41]}$ and ceftazidime/avibactam ${ }^{[42]}$ previously, two types of variations in acrR-gene was observed in the present study: one was the genotypic variation in non-ST11 isolates representing geographical difference and the other was acrR-mutation, IS5-insertion-inactivation, shown by almost all isolates of ST11, which 
was different from frameshift-deletion in genetically unrelated $K$. pneumoniae isolates from Singapore hospitals ${ }^{[26]}$. Our finding gave an exciting evidence to decipher the molecular basis of $A c r A B$ overexpression as a resistance mechanism, supporting the ST11 as the predominant clone of MDR-KP infections in China ${ }^{[43]}$. Furthermore, OmpK35 and OmpK36, two major co-regulated OM proteins, provided a channel that allows the selective uptake of essential nutrients and multiple antibiotics. Porin deficiency is a widespread phenomenon in $K$. pneumoniae. OmpK36 absence has been related to antimicrobial resistance but also decreased fitness and diminished virulence ${ }^{[44,45]}$; OmpK35, unnecessary for pathogenesis and resistance ${ }^{[44]}$, is commonly absent from ESBL- ${ }^{[46]}$ or KPC- ${ }^{[42,47]}$ producers. All seven ciprofloxacin-susceptible isolates in this study kept the coding sequences of both porins integral. However, isolates of ST11 (wzi64, wzi209) and ST15 (wzi19) had frameshift disruptions in OmpK35, accompanied with a specific L3-modification (ins aa134-135 GD) in OmpK36, which has been reported to contribute to the emergence of carbapenem resistance $e^{[28,46,47,48]}$, suggesting as a solution to the fitness cost imposed by OmpK36 loss. The specific OmpK35/OmpK36 profiles formed a trade-off between antimicrobials permeability and nutritional competence, which led high-risk MDR clones to epidemic success. Altogether, reduced influx through porin channels often acted in concert with increased efflux by pumps to confer an antibiotic cross-resistance phenotype in the majority of MDR-KP isolates.

In China, the resistance rates of $K$. pneumoniae to four commonly used antimicrobials, ciprofloxacin (34.0\%), ceftazidime (34.7\%), piperacillin-tazobactam (26.3\%), and amikacin (15.7\%), had markedly increased since 2015 to 2020 following a 2005-2014 decline trend, which was consistent with the marked increase in CRKPs from 2005 (3.0\%) to 2020 (23.2\%), while ESBL production (cefotaxime-resistance) fluctuating at about $30-40 \%$ meanwhile ${ }^{[8]}$. PMQR $(n=32,94.1 \%)$ are often associated with co-resistance to other antimicrobials owing to the co-localization of resistance-conferring genes on the same plasmid or isolate ${ }^{[49]}$, a critical feature that fosters the horizontal dissemination of MDR partly explaining the less optimistic resistance trend since 2015. Thirty CRKP isolates herein carried at least one or more commonly both of the two predominant $\beta$-lactamase genotypes, KPC-2-type carbapenemase and CTX-M-type ESBL ${ }^{[50,51]}$. Acquisition of class A 
256 (KPC-2, KPC-3) and B (New Delhi metallo- $\beta$-lactamase (NDM)-1) carbapenemases $^{[51]}$ predominantly

257 contribute to carbapenem-resistance mechanisms; production of ESBL (A) and/or AmpC (C)

$258 \beta$-lactamases in combination with acquired OM impermeability owing to mutation-derived

259 modifications in major porins ${ }^{[52]}$ and/or AcrR were also contributors. In view of the extremely high

260 resistance rate of CRKP to ciprofloxacin $\left(93.2 \%\right.$, CHINET, 2020) ${ }^{[8]}$, co-resistance to quinolones and

261 carbapenems was observed in 26 out of 30 CRKPs, leaving few alternatives to first-line agents for the

262 treatment of serious nosocomial infections. Usually, combination therapy based on tigecycline or

263 polymyxin coupled with carbapenems, aminoglycosides, or fosfomycin was proposed ${ }^{[53]}$. The

enhanced efficacy of a carbapenem-containing regimen against CPKPs (MICs $\leq 4 \mathrm{mg} / \mathrm{L}$ ) when administered at high-dose/prolonged infusion ${ }^{[54]}$ kept carbapenems a place in the treatment of CPKP infections. Notably, the occurrence of ArmA and RmtB in isolates of ST15 (wzi24) and ST11 (wzi64/wzi 209), respectively, conferred high level resistance to almost all clinically important aminoglycosides by methylation of ribosomal target ${ }^{[24]}$ further complicating therapy. Unlike $16 \mathrm{~S}$ rRNA methylases, AMEs vary in their substrate ranges, e.g. AAC $\left(6^{\prime}\right)-\mathrm{lb}$-cr was active on gentamicin but not amikacin in isolates ST15 (wzi19). Fortunately, in the absence of QRDR-mutations, CRKPs might remain susceptible to ciprofloxacin, e.g. isolates (ST13, ST65) from sputum in ND, a phenomenon once underestimated that rendered quinolones a potential complementation to the limited antibiotic options against CRKP infections. Hence, combination or monotherapy based on quinolones was commonly used to manage adult patients with MDR-KP induced HAP or VAP ${ }^{[55]}$. Recently, the compound ceftazidime/avibactam was introduced in China to effectively fight against CRKPs carrying all combinations of $\beta$-lactamases (class $A, C$ and $D)^{[42,51,56]}$, with little effect on the emerging KPC-2 and NDM-1-coproducers characterized by high stability, non-inferior fitness and transferability among patients ${ }^{[57]}$. Here, such NDM co-producing CRKP of ST13 concomitantly acquired additional $\beta$-lactamases of other types, evolving into a super-producer of quadruple enzymes with potentially higher $\beta$-lactam hydrolysis activity. Isolates of ST65 (wzi72-K2) having unique efflux pumps (oqxA10B6, potl-v3) shared the same 
become a common pathogen of VAP in mechanically ventilated patients in China ${ }^{[32,58]}$. These findings highlighted an emerging and escalating threat of CRKPs to healthcare in the clinical settings.

Nucleotide sequence-based typing methods, $\mathrm{MLST}^{[10]}$ and wzi-genotyping ${ }^{[11]}$, can provide unambiguous and portable data useful for epidemiological studies. We hereby observed a strong correlation of $o m p K 36$ variants with specific lineages of $K$. pneumoniae isolates, as shown by the $\mathrm{ST}^{[59]}$ but also wzi-allele, suggesting that OmpK36 has a higher evolutionary rate. Mutational changes in adaptation to host colonization/infection and antimicrobial pressure are frequently selected, so virulence (e.g. capsule, efflux pump, and porin) and resistance is polymorphic. High-risk epidemic clones employed these mechanisms to change their ability to persist better in the environment and human populations. The wzi-encoding surface protein involved in capsule attachment to the $\mathrm{OM}^{[60]}$ was strongly associated with specific $\mathrm{K}$ antigens $(\mathrm{K} 1-\mathrm{K} 78)^{[11]}$. Age- and site-specific distribution of wzi-alleles were observed in isolates of ST11 (infant-wzi209, adult-wzi64) and ST15 (ICU-wzi24, ND-wzi19), respectively. Besides allelic mutations in genes of wzi, oqxAB, and $a c r R$, multidirectional evolutionary trend of major porin genotypes was presented in isolate JSK7111 (ST218), another trade-off between fitness cost and heterogeneity of antibiotic exclusion by allowing coexistence of mutated and non-mutated versions of porins before reaching a stable state for a new variant. In a fatal nosocomial outbreak, the transmission of isolates ST15 (wzi24-K24) among aged inpatients in ICU induced new ompK36 variants co-circulating, despite all holding single integral ompK35. Compared to the epidemic ompK36-genotype (036K7233) in China in the last decade, the sudden predominance of emerging variant 036K7106 was possibly associated with serious infection and poor outcome, accompanied with the emergence of KPC-3 and CTX-M-15 co-producing CRKPs, evidence that the S7F-substitution increased the infectivity and virulence of isolates ST15. Ineffectiveness of QRDR-mutation in a CTX-M-15 producer susceptible to both ciprofloxacin and carbapenem was possibly attributable to a pre-epidemic ompK36 intermediate (036K7340) identical to only 3 Lithuania isolates from a global survey ${ }^{[52]}$. Furthermore, OmpK36 phenotype could influence carbapenem susceptibility in KPC-producers, either making them go unrecognized in routine susceptibility testing, e.g. isolate JSK7341 (potl-v3), or correlating enhanced resistance to $\beta$-lactams with a special OmpK36 
309 configuration in ST11 clone ${ }^{[28,41,42]}$. Combined, a shift in ompK36 genotypes seemed to be also

310 involved in the reduction of OM permeability, resulting to changed antibiogram or attenuated

311 virulence. An ompK36-targeted PCR method can cost-effectively distinguish major high-risk resistance

312 and virulence clones of $K$. pneumoniae, but lacking the specificity for screening of epidemic $\mathrm{ST}^{[61]}$.

313 Here, we preliminarily analyzed the exploitability of a genotyping framework based on ompK36-alleles

314 of the representative $K$. pneumoniae worldwide. Future work remains to be done to establish a

315 multiple-tiered classification system for the better understanding of how porin heterogeneity and

316 trade-offs might affect the MDR phenotype and infection outcome of isolates.

\section{Conclusions}

In conclusion, accumulation of mutations of three categories and acquisition of resistance-conferring genes contribute to quinolone resistance in clinical MDR-KP isolates. Under multiple selective pressures, QRDR-mutations are sufficient but not absolutely necessary for acquiring quinolone resistance, further promoted by spread of PMQR determinants, and frequently co-regulated by mutations in AcrR and/or OmpK36; all closely correlated with combined-resistance to multiple antimicrobials, thereby necessitating and strengthening continuing epidemiologic surveillance, strict infection control measures and restrictive antibiotic stewardship.

\section{Supplementary Information}

The online version contains supplementary material

327 Additional file 1: Table S1. Primer sequences used for PCR amplification of resistant-associated genes for K. pneumoniae.

\section{Abbreviations}

aa: amino acid; ABC: ATP-binding cassette; AME: aminoglycoside-modifying enzyme; bp: base pair(s);

CRKP: carbapenem-resistant Klebsiella pneumoniae; Da: dalton(s); ESBL: extended-spectrum 
ins aa 134-135 GD: insertions encoding glycine and aspartic acid at aa positions 134 and 135; IS: insertion sequence; K: capsular; KPC: Klebsiella pneumoniae carbapenemase; MDR: multidrug resistant or multidrug resistance; MIC: minimum inhibitory concentration; MLST: multilocus sequence typing; ND: neurosurgery department; NDM, New Delhi metallo- $\beta$-lactamase; nt: nucleotide(s); OM: outer membrane; ORF: open reading frame; P: probability; PCR: polymerase chain reaction; PMQR: plasmid-mediated quinolone resistance; QRDR: quinolone resistance-determining region; RND: resistance-nodulation-division; ST: sequence type; VAP: ventilator-associated pneumonia.

\section{Acknowledgments}

The authors thank all of the people involved in clinical and molecular epidemiological investigation of this study.

\section{Authors' contributions}

YW and H-ZL conceived and designed the study. YW collected the isolates and performed the experiments. G-PC and J-AZ participated in the data collection. YW and G-PC analyzed the data and drafted the manuscript. H-ZL, J-AZ, and X-GX revised the manuscript critically for intellectual content. All authors read and gave final approval of the manuscript.

\section{Funding}

This work was funded by the grants from Jinshan District Health and Family Planning Commission of Shanghai Municipality, China (JSKJ-KTMS-2015-04) and from Clinical Research Special Project in Health Industry, Shanghai Municipal Health Commission of China (201940361). The funders had no role in the design and conduct of the study; collection, management, analysis, and interpretation of the data; preparation, review, or approval of the manuscript; and decision to submit the manuscript for publication.

\section{Availability of data and materials}

All materials and data analyzed during this study are contained within the manuscript. 


\section{Ethics approval and consent to participate}

358 The study protocol was approved by the Medical Ethics Committee of Jinshan Hospital, Fudan

359 University. Informed consent was not obtained due to the retrospective and anonymous nature of the 360 study.

\section{Consent for publication}

362 Not applicable.

\section{Competing interests}

364 The authors declare that they have no competing interests.

\section{Author details}

$366{ }^{1}$ Research Center for Clinical Medicine, Jinshan Hospital, Fudan University, Shanghai, China;

$367 \quad{ }^{2}$ Department of Surgery, Jinshan Hospital, Fudan University, Shanghai, China; ${ }^{3}$ Department of Internal 368 Medicine, Zhoupu Hospital, Shanghai Jiankang Medical University, Shanghai, China; ${ }^{4}$ Institute of 369 Antibiotics, Huashan Hospital, Fudan University, Shanghai, China; ${ }^{5}$ Department of infections and 370 immunity, Shanghai Public Health Clinical Center, Fudan University, Shanghai, China

\section{$371 \quad$ References}

1. Hooper DC. Bacterial topoisomerases, anti-topoisomerases, and anti-topoisomerase resistance. Clin 373 Infect Dis. 1997; 27(Suppl_1):S54-63.

374 2. Jacoby GA, Strahilevitz J, Hooper DC. Plasmid-mediated quinolone resistance. Microbiol Spectr. $375 \quad 2014 ; 2(5): 10.1128$.

376 3. Chevalier J, Pagès JM, Eyraud A, Malléa M. Membrane permeability modifications are involved in antibiotic resistance in Klebsiella pneumoniae. Biochem Biophys Res Commun. 2000; 274(2): 496-9. 
4. Poole K. Efflux-mediated resistance to fluoroquinolones in gram-negative bacteria. Antimicrob Agents Chemother. 2000; 44(9): 2233-41.

5. Stewardson AJ, Marimuthu K, Sengupta S, Allignol A, El-Bouseary M, Carvalho MJ, et al. Effect of carbapenem resistance on outcomes of bloodstream infection caused by Enterobacteriaceae in low-income and middle-income countries (PANORAMA): a multinational prospective cohort study. Lancet Infect Dis. 2019; 19(6): 601-10.

6. Avendano EE, Raman G, Chan J, and McCann E. Burden of carbapenem non-susceptible infections in high-risk patients: systematic literature review and meta-analysis. Antimicrob Resist Infect Control. 2020; 9: 193.

7. CHINET data cloud. Surveillance results of antimicrobial resistance in 2020. China Antimicrobial Surveillance Network (CHINET) [EB/OL]. [2021-02-22]. http://www.chinets.com/Documents.

8. The Center for Disease Dynamics, Economics \& Policy. ResistanceMap: Antibiotic resistance. CDDEP, Washington, D.C., USA, and New Delhi, India, 2017.

9. Clinical and Laboratory Standards Institute. Performance Standards for Antimicrobial Susceptibility Testing: Twenty-Seventh Informational Supplement M100-S27. CLSI, Wayne, PA, USA, 2017.

10. Diancourt L, Passet V, Verhoef J, Grimont PA, Brisse S. Multilocus sequence typing of Klebsiella pneumonia nosocomial isolates. J Clin Microbiol. 2005; 43(8): 4178-82.

11. Brisse $\mathrm{S}$, Passet V, Haugaard AB, Babosan A, Kassis-Chikhani N, Struve C, et al. Wzi gene sequencing, a rapid method to determine the capsular type of Klebsiella strains. J Clin Microbiol. 2013; 51(12): 4073-8.

12. Lavilla S, González-López JJ, Sabaté M, García-Fernández A, Larrosa MN, Bartolomé RM, et al. Prevalence of qnr genes among extended-spectrum $\beta$-lactamase-producing enterobacterial isolates in Barcelona, Spain. J Antimicrob Chemother. 2008; 61(2): 291-5. 
401 13. Wu JJ, Ko WC, Tsai SH, Yan JJ. Prevalence of plasmid-mediated quinolone resistance determinants 402 QnrA, QnrB, and QnrS among clinical isolates of Enterobacter cloacae in a Taiwanese hospital. 403 Antimicrob Agents Chemother. 2007; 51(4): 1223-7.

404 14. Wang $M H$, Guo GL, $X u$ XG, Wang $X Y, Y e X Y, W u S$, et al. New plasmid-mediated quinolone 405 resistance gene, gnrC, found in a clinical isolate of Proteus mirabilis. Antimicrob Agents Chemother. $406 \quad 2009 ; 53(5): 1892-7$.

407 15. Cavaco LM, Hasman H, Xia S, Aarestrup FM. qnrD, a novel gene conferring transferable quinolone 408 resistance in Salmonella enterica Serovar Kentucky and Bovismorbificans strains of human origin. 409 Antimicrob Agents Chemother. 2009; 53(2): 603-8.

16. Park $\mathrm{CH}$, Robicsek A, Jacoby GA, Sahm D, Hooper DC. Prevalence in the United States of $a a c\left(6^{\prime}\right)-I b-c r$ encoding a ciprofloxacin-modifying enzyme. Antimicrob Agents Chemother. 2006; 50(11): 3953-5.

17. Ma JY, Zeng ZL, Chen ZL, Xu XG, Wang XY, Deng YT, et al. High prevalence of plasmid-mediated 414 quinolone resistance determinants $q n r$, $a a c\left(6^{\prime}\right)-I b-c r$, and $q e p A$ among ceftiofur-resistant 415 Enterobacteriaceae isolates from companion and food-producing animals. Antimicrob Agents 416 Chemother. 2009; 53(2): 519-24.

417 18. Kim HB, Wang MH, Park CH, Kim EC, Jacoby GA, Hooper DC. oqxAB encoding a multidrug efflux 418 pump in human clinical isolates of Enterobacteriaceae. Antimicrob Agents Chemother. 2009; 53(8): $419 \quad 3582-4$

420 19. Yuan JY, Xu XG, Guo QL, Zhao X, Ye XY, Guo Y, et al. Prevalence of the oqxAB gene complex in 421 Klebsiella pneumoniae and Escherichia coli clinical isolates. J Antimicrob Chemother. 2012; 67(7): $1655-9$. 
20. Hoffmann H, Stürenburg E, Heesemann J, Roggenkamp A. Prevalence of extended-spectrum $\beta$-lactamases in isolates of the Enterobacter cloacae complex from German hospitals. Clin Microbiol Infect. 2006; 12(4): 322-30.

21. Pérez-Pérez FJ, Hanson ND. Detection of plasmid-mediated AmpC $\beta$-lactamase genes in clinical isolates by using multiplex PCR. J Clin Microbiol. 2002; 40(6): 2153-62.

22. Bradford PA, Bratu S, Urban C, Visalli M, Mariano N, Landman D, et al. Emergence of carbapenem-resistant Klebsiella species possessing the class A carbapenem-hydrolyzing KPC-2 and inhibitor-resistant TEM-30 ß-lactamases in New York city. Clin Infect Dis. 2004; 39(1): 55-60.

23. Poirel L, Walsh TR, Cuvillier V, Nordmann P. Multiplex PCR for detection of acquired carbapenemase genes. Diagn Microbiol Infect Dis. 2011; 70(1): 119-23.

24. Yan JJ, Wu JJ, Ko WC, Tsai SH, Chuang CL, Wu HM, et al. Plasmid-mediated $16 \mathrm{~S}$ rRNA methylases conferring high-level aminoglycoside resistance in Escherichia coli and Klebsiella pneumoniae isolates from two Taiwanese hospitals. J Antimicrob Chemother. 2004; 54(6): 1007-12.

25. Kaczmarek FM, Dib-Hajj F, Shang W, Gootz TD. High-level carbapenem resistance in a Klebsiella pneumoniae clinical isolate is due to the combination of $b / a_{\mathrm{ACT}-1} \beta$-lactamase production, porin OmpK35/36 insertional inactivation, and down-regulation of the phosphate transport porin PhoE. Antimicrob Agents Chemother. 2006; 50(10): 3396-406.

26. Schneiders T, Amyes SG, Levy SB. Role of AcrR and RamA in fluoroquinolone resistance in clinical Klebsiella pneumoniae isolates from Singapore. Antimicrob Agents Chemother. 2003; 47(9): 2831-7.

27. Tamura K, Stecher G, Tamura K. MEGA7: molecular evolutionary genetics analysis version 7.0 for bigger datasets. Mol Biol Evol. 2016; 33(7): 1870-4.

28. Zhang Y, Jiang XF, Wang YY, Li G, Tian YR, Liu H, et al. Contribution of $\beta$-Lactamases and porin proteins OmpK35 and OmpK36 to carbapenem resistance in clinical isolates of KPC-2-producing Klebsiella pneumonia. Antimicrob Agents Chemother. 2014; 58(2): 1214-7. 
29. Guo Y, Zhou H, Qin L, Pang ZZ, Qin T, Ren HY, et al. Frequency, antimicrobial resistance and genetic diversity of Klebsiella pneumoniae in food samples. PLoS One. 2016; 11(4): e0153561.

30. Chia PY, Sengupta S, Kukreja A, Ponnampalavanar SSL, Ng OT, and Marimuthu K. The role of hospital environment in transmissions of multidrug-resistant gram-negative organisms. Antimicrob Resist Infect Control. 2020; 9(1): 29.

31. Calbo E, Freixas N, Xercavins M, Riera M, Nicolás C, Monistrol O, et al. Foodborne nosocomial outbreak of SHV1 and CTX-M-15-producing Klebsiella pneumoniae: epidemiology and control. Clin Infect Dis. 2011; 52(6): 743-9.

32. Gu DX, Dong N, Zheng ZW, Lin D, Huang $\mathrm{M}$, Wang LH, et al. A fatal outbreak of ST11 carbapenem-resistant hypervirulent Klebsiella pneumoniae in a Chinese hospital: a molecular epidemiological study. Lancet Infect Dis. 2018; 18(1): 37-46.

33. Drlica K. The mutant selection window and antimicrobial resistance. J Antimicrob Chemother. 2003; 52(1): 11-7.

34. Hansen LH, Johannesen E, Burmølle M, Sørensen AH, Sørensen SJ. Plasmid-encoded multidrug efflux pump conferring resistance to olaquindox in Escherichia coli. Antimicrob Agents Chemother. 2004; 48(9): 3332-7.

35. Perez F, Rudin SD, Marshall SH, Coakley P, Chen L, Kreiswirth BN, et al. OqxAB, a quinolone and qlaquindox efflux pump, is widely distributed among multidrug-resistant Klebsiella pneumoniae isolates of human origin. Antimicrob Agents Chemother. 2013; 57(9): 4602-3.

36. Rees $\mathrm{DC}$, Johnson $\mathrm{E}$, Lewinson $\mathrm{O}$. ABC transporters: the power to change. Nat Rev Mol Cell Biol. 2009; 10(3): 218-27.

37. Perez-Leal O, Merali S. Regulation of polyamine metabolism by translational control. Amino Acids. $2012 ; 42(2-3): 611-7$. 
38. Sato T, Yokota S, Uchida I, Okubo T, Usui M, Kusumoto M, et al. Fluoroquinolone resistance mechanisms in an Escherichia coli isolate, HUE1, without quinolone resistance-determining region mutations. Front Microbiol. 2013; 4: 125.

39. Bialek-Davenet S, Lavigne JP, Guyot K, Mayer N, Tournebize R, Brisse S, et al. Differential contribution of $\mathrm{Acr} A \mathrm{~B}$ and OqxAB efflux pumps to multidrug resistance and virulence in Klebsiella pneumoniae. J Antimicrob Chemother. 2015; 70(1): 81-8.

40. Oethinger M, Kern WV, Jellen-Ritter AS, McMurry LM, Levy SB. Ineffectiveness of topoisomerase mutations in mediating clinically significant fluoroquinolone resistance in Escherichia coli in the absence of the AcrAB efflux pump. Antimicrob Agents Chemother. 2000; 44(1): 10-3.

41. Landman D, Bratu S, Quale J. Contribution of OmpK36 to carbapenem susceptibility in KPC-producing Klebsiella pneumonia. J Med Microbiol. 2009; 58(Pt10): 1303-8.

42. Shen $\mathrm{Z}$, Ding $\mathrm{B}$, Ye M, Wang $\mathrm{P}, \mathrm{Bi} \mathrm{Y}, \mathrm{Wu} \mathrm{S}$, et al. High ceftazidime hydrolysis activity and porin OmpK35 deficiency contribute to the decreased susceptibility to ceftazidime/avibactam in KPC-producing Klebsiella pneumonia. J Antimicrob Chemother. 2017; 72(7): 1930-6.

43. Qi Y, Wei Z, Ji S, Du X, Shen P, Yu Y. ST11, the dominant clone of KPC-producing Klebsiella pneumoniae in China. J Antimicrob Chemother. 2011; 66(2): 307-12.

44. Fajardo-Lubián A, Ben Zakour NL, Agyekum A, Qi Q, Iredell JR. Host adaptation and convergent evolution increases antibiotic resistance without loss of virulence in a major human pathogen. PLoS Pathog. 2019; 15(3): e1007218.

45. Wong JLC, Romano M, Kerry LE, Kwong HS, Low WW, Brett SJ, et al. OmpK36-mediated carbapenem resistance attenuates ST258 Klebsiella pneumoniae in vivo. Nat Commun. 2019; 10(1): 3957. 
46. García-Fernández A, Miriagou V, Papagiannitsis CC, Giordano A, Venditti M, Mancini C, et al. An ertapenem-resistant extended-spectrum-\{beta\}-lactamase-producing Klebsiella pneumoniae clone carries a novel OmpK36 porin variant. Antimicrob Agents Chemother. 2010; 54(10): 4178-84.

47. Clancy CJ, Chen L, Hong JH, Cheng S, Hao B, Shields RK, et al. Mutations of the ompK36 porin gene and promoter impact responses of sequence type 258, KPC-2-producing Klebsiella pneumoniae strains to doripenem and doripenem-colistin. Antimicrob Agents Chemother. 2013; 57(11): 5258-65.

48. Wilson WR, Kline EG, Jones CE, Morder KT, Mettus RT, Doi Y, et al. Effects of KPC variant and porin genotype on the in vitro activity of meropenem-vaborbactam against carbapenem-resistant Enterobacteriaceae. Antimicrob Agents Chemother. 2019; 63(3): e02048-18.

49. Ramirez MS, Iriarte A, Reyes-Lamothe R, Sherratt DJ, Tolmasky ME. Small Klebsiella pneumoniae plasmids: neglected contributors to antibiotic resistance. Front Microbiol. 2019; 10: 2182.

50. Chen S, Hu F, Xu X, Liu Y, Wu W, Zhu D, et al. High prevalence of KPC-2-type carbapenemase coupled with CTX-M-type extended-spectrum $\beta$-lactamases in carbapenem-resistant Klebsiella pneumoniae in a teaching hospital in China. Antimicrob Agents Chemother. 2011; 55(5): 2493-4.

51. Han R, Shi Q, Wu S, Yin D, Peng M, Dong D, et al. Dissemination of carbapenemases (KPC, NDM, OXA-48, IMP, and VIM) among carbapenem-resistant Enterobacteriaceae isolated from adult and children patients in China. Front Cell Infect Microbiol. 2020; 10: 314.

52. Wise MG, Horvath E, Young K, Sahm DF, Kazmierczak KM. Global survey of Klebsiella pneumoniae major porins from ertapenem non-susceptible isolates lacking carbapenemases. J Med Microbiol. 2018; 67(3): 289-95.

53. Chinese XDR Consensus Working Group; Guan X, He L, Hu B, Hu J, Huang X, et al. Laboratory diagnosis, clinical management and infection control of the infections caused by extensively drug-resistant Gram-negative bacilli: a Chinese consensus statement. Clin Microbiol Infect. 2016; 22(Suppl_1): S15-25. 
54. Daikos GL, Markogiannakis A. Carbapenemase-producing Klebsiella pneumoniae: (when) might we still consider treating with carbapenems? Clin Microbiol Infect. 2011; 17(8): 1135-41.

55. Torres A, Niederman MS, Chastre J, Ewig S, Fernandez-Vandellos P, Hanberger H, et al. International ERS/ESICM/ESCMID/ALAT guidelines for the management of hospital-acquired pneumonia and ventilator-associated pneumonia. Eur Respir J. 2017; 50(3): 1700582.

56. Kazmierczak KM, de Jonge BLM, Stone GG, Sahm DF. In vitro activity of ceftazidime/avibactam against isolates of Enterobacteriaceae collected in European countries: INFORM global surveillance 2012-15. J Antimicrob Chemother. 2018; 73(10): 2782-8.

57. Gao H, Liu Y, Wang R, Wang Q, Jin L, Wang H. The transferability and evolution of NDM-1 and KPC-2 co-producing Klebsiella pneumoniae from clinical settings. EBioMedicine. 2020; 51: 102599.

58. Yan Q, Zhou M, Zou M, Liu WE. Hypervirulent Klebsiella pneumoniae induced ventilator-associated pneumonia in mechanically ventilated patients in China. Eur J Clin Microbiol Infect Dis. 2016; 35(3): 387-96.

59. Papagiannitsis CC, Giakkoupi P, Kotsakis SD, Tzelepi E, Tzouvelekis LS, Vatopoulos AC, et al. OmpK35 and OmpK36 porin variants associated with specific sequence types of Klebsiella pneumoniae. J Chemother. 2013; 25(4): 250-4.

60. Rahn A, Beis K, Naismith JH, Whitfield C. A novel outer membrane protein, Wzi, is involved in surface assembly of the Escherichia coli K30 Group 1 Capsule. J Bacteriol. 2003; 185(19): 5882-90.

61. Yan JJ, Zheng PX, Wang MC, Tsai SH, Wang LR, Wu JJ. Allocation of Klebsiella pneumoniae bloodstream isolates into four distinct groups by ompK36 typing in a Taiwanese university hospital. J Clin Microbiol. 2015; 53(10): 3256-63. 
Figure 1. Phylogenetic analysis based on the coding sequences of ompK36 genes for K. pneumoniae isolates. The dendrogram was constructed using neighbor-joining method by MEGA program version 7.0. Bootstrap resampling (1000 replications) was used, and bootstrap values $\geq 70 \%$ were shown. Scale bar represents nt substitutions per site. Reference strains were named in reference to the accession-number, strain name, country of origin, isolation-year, and MLST. $\star$ denotes $K$. pneumoniae isolates in the present study (GenBank accession number MK341466-MK341478) with its common name and ST shown in bold. The ompK36 for a Raoultella planticola isolate (CP026047) was taken as an out-group. The red box indicates the phylogenetic cluster position of isolates ST11 (Lineage IV). 
Figure 1.

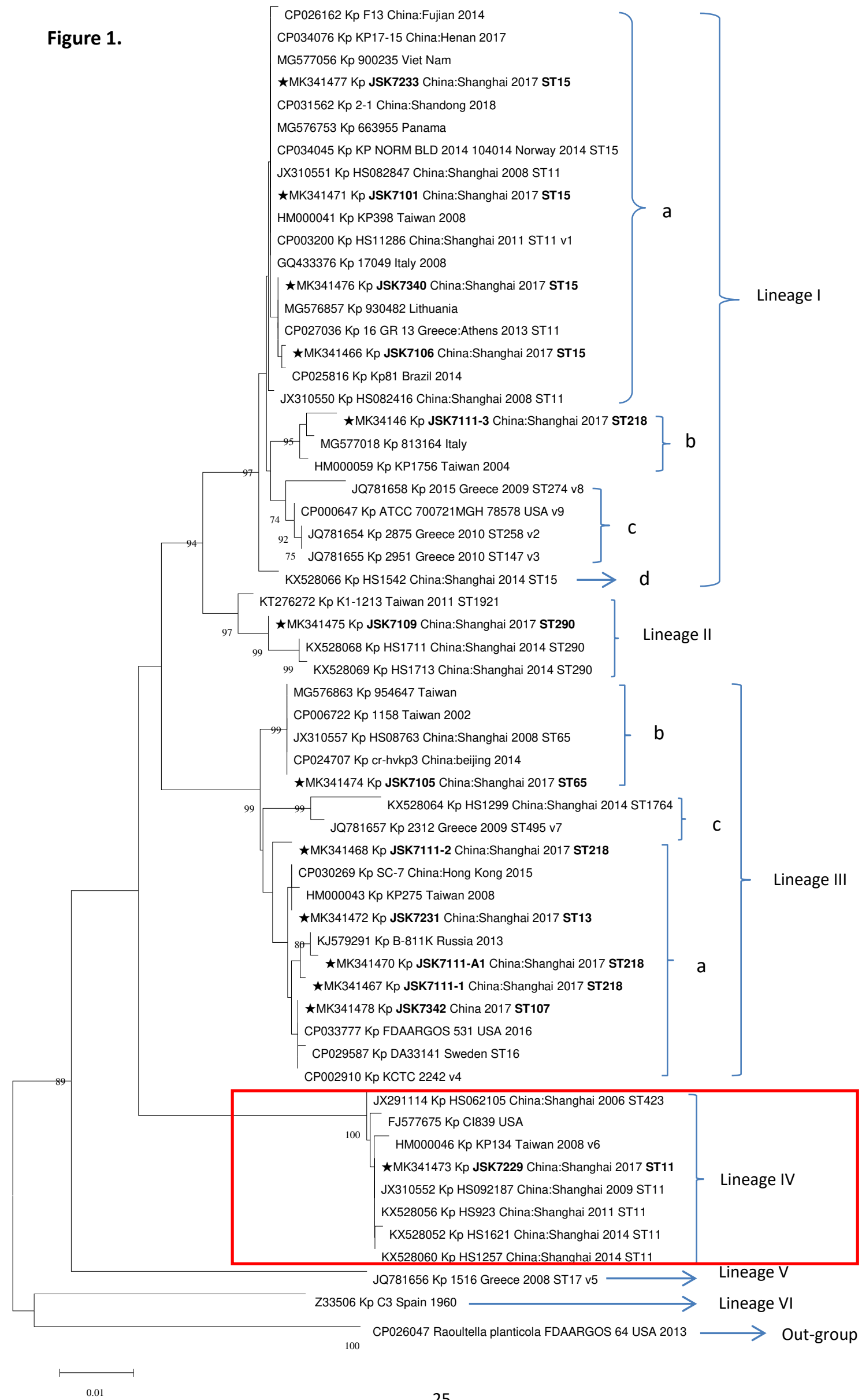
CP034076 Kp KP17-15 China:Henan 2017 G577056 Kp 900235 Viet Nam CP031562 Kp 2-1 China:Shandong 2018 53 Kp 663955 Panama JX310551 Kp HS082847 China:Shanghai 2008 ST1 CP003200 Kp HS11286 China:Shanghai 2011 ST11 v MG576857 Kp 930482 Lithuania

CP027036 Kp 16 GR 13 Greece:Athens 2013 ST1 KK31466 Kp JSK7106 China:Shanghai 2017 ST15 HS082416 China:S

Lineage II

JX310557 Kp HS08763 China:Shanghai 2008 ST65

CP024707 Kp cr-hvkp3 China:beijing 2014 KP JSK7105 China:Shanghai 2017 ST65

CP026162 Kp F13 China:Fujian 2014 
Table 1. Characterization of phenotypic and genotypic resistance of $34 \mathrm{~K}$. pneumonia isolates from nosocomial infections

\begin{tabular}{|c|c|c|c|c|c|c|c|c|c|c|}
\hline \multirow{2}{*}{ Isolate } & \multirow{2}{*}{ Specimen } & \multirow{2}{*}{ Susceptibility profiles ${ }^{a}$} & \multirow{2}{*}{$\mathrm{PMQR}^{\mathrm{b}}$} & \multicolumn{2}{|c|}{ QRDR mutations } & \multirow{2}{*}{ Other resistance genes } & \multirow{2}{*}{ ST } & \multirow{2}{*}{$w z i-K$ type $^{c}$} & \multirow{2}{*}{ ompK $36^{d}$} & \multirow{2}{*}{ ompK35 ${ }^{\mathrm{d}}$} \\
\hline & & & & GyrA & ParC & & & & & \\
\hline JSK7101 & Sputum & AMK, SXT, FOF & $a a c\left(6^{\prime}\right)-I b-c r$, oqxA8B20 & S83F; D87A & s80I & CTX-M-15,TEM-1,SHV-1,KPC-2 & 15 & wzi19-K19 & $\mathrm{la}, 036 \mathrm{~K} 7101^{\mathrm{iGD}}$ & O35K7101 ${ }^{\mathrm{FI}}$ \\
\hline JSK7102 & Sputum & AMK, SXT, FOF & $\operatorname{aac}\left(6^{\prime}\right)-I b-c r$, oqxA8B20 & S83F; D87A & s80I & CTX-M-15,TEM-1,SHV-1 & 15 & wzi19-K19 & O36K7101 ${ }^{\text {iGD }}$ & O35K7101 ${ }^{\mathrm{FSI}}$ \\
\hline JSK7108 & blood & AMK, SXT, FOF & $\operatorname{aac}\left(6^{\prime}\right)-I b-c r$, oqxA8B20 & S83F; D87A & $\mathrm{S} 80 \mathrm{l}$ & CTX-M-15,TEM-1 & 15 & wzi19-K19 & O36K7101 ${ }^{\mathrm{iGD}}$ & O35K $7101^{\mathrm{FSI}}$ \\
\hline JSK7112 & Sputum & AMK, SXT, FOF & $\operatorname{aac}\left(6^{\prime}\right)-I b-c r$, oqxA8B20 & S83F; D87A & $\mathrm{s} 80 \mathrm{O}$ & CTX-M-15,TEM-1,SHV-1 & 15 & wzi19-K19 & O36K7101 ${ }^{\text {iGD }}$ & O35K7101 ${ }^{\mathrm{FI}}$ \\
\hline JSK7120 & Sputum & AMK, SXT, FOF & $\operatorname{aac}\left(6^{\prime}\right)-I b-c r$, oqxB20 & S83F; D87A & s80I & СТX-M-15 & 15 & wzi19-K19 & O36K7101 ${ }^{\mathrm{iGD}}$ & 035K7106 \\
\hline JSK7223 & Sputum & AMK, SXT, FOF & $a a c\left(6^{\prime}\right)-I b-c r, o q \times B 20, p o t l-v 2$ & S83F; D87A & $\mathrm{s} 80 \mathrm{l}$ & CTX-M-15,KPC-2 & 15 & wzi19-K19 & O36K7101 ${ }^{\text {iGD }}$ & O35K7101 ${ }^{\mathrm{FSI}}$ \\
\hline JSK7224 & Sputum & AMK, SXT & $\operatorname{aac}\left(6^{\prime}\right)-|l b-c r, o q \times B 20, p o t|-\mathrm{v} 2$ & S83F; D87A & s80I & CTX-M-15,KPC-2 & 15 & wzi19-K19 & O36K7101 ${ }^{\text {iGD }}$ & O35K7101 ${ }^{\mathrm{FI}}$ \\
\hline JSK7233 ${ }^{\times}$ & Urine & NTA & oqxA8B20, potl-v2 & S83F; D87A & s80I & CTX-M-15,TEM-1,SHV-1,KPC-2,armA & 15 & wzi24-K24 & la, 036K7233 & 035K7106 \\
\hline JSK7338 & Sputum & FOF & oqxA8B20 & S83F; D87A & $\mathrm{S} 80 \mathrm{l}$ & CTX-M-15,TEM-1,SHV-1,KPC-2,armA & 15 & wzi24-K24 & O36K7233 & O35K7106 \\
\hline JSK7340 & Sputum & CIP, TZP, IPM, MEM, FOF & oqxA8B20, potl-v2 & S83F; D87A & $\mathrm{s} 80 \mathrm{O}$ & CTX-M-15,TEM-1,SHV-1,armA & 15 & wzi24-K24 & la, 036K7340 & O35K7106 \\
\hline JSK7106* & Sputum & FOF & oqxB20 & S83F; D87A & S80I & CTX-M-15, armA & 15 & wzi24-K24 & la, O36K7106 & O35K7106 \\
\hline JSK7107* & Sputum & FOF & NPA & S83F; D87A & $\mathrm{s} 80 \mathrm{I}$ & CTX-M-15,TEM-1,armA & 15 & wzi24-K24 & 036K7106 & O35K7106 \\
\hline $\operatorname{JSK7110^{\wedge }\times }$ & Sputum & FOF & oqxA8B20 & S83F; D87A & $\mathrm{s} 80 \mathrm{l}$ & CTX-M-15,TEM-1,SHV-1,armA & 15 & wzi24-K24 & 036K7106 & O35K7106 \\
\hline JSK7119* & Sputum & FOF & oqxB20 & S83F; D87A & $\mathrm{s} 80 \mathrm{l}$ & CTX-M-15 & 15 & wzi24-K24 & O36K7106 & O35K7106 \\
\hline JSK7225 & Sputum & FOF & oqxB20, potl-v2 & S83F; D87A & $\mathrm{S} 80 \mathrm{l}$ & CTX-M-15,KPC-3, armA & 15 & wzi24-K24 & O36K7106 & O35K7106 \\
\hline $\operatorname{JSK} 7345^{\wedge^{\times}}$ & Urine & FOF & oqxA8B20 & S83F; D87A & S80I & $\begin{array}{l}\text { CTX-M-15, } \\
\text { KPC-3 variant }(\mathrm{C} 68 \mathrm{~A} \rightarrow \mathrm{T} 23 \mathrm{~N})\end{array}$ & 15 & wzi24-K24 & O36K7106 & O35K7106 \\
\hline
\end{tabular}


Table 1. (Continued)

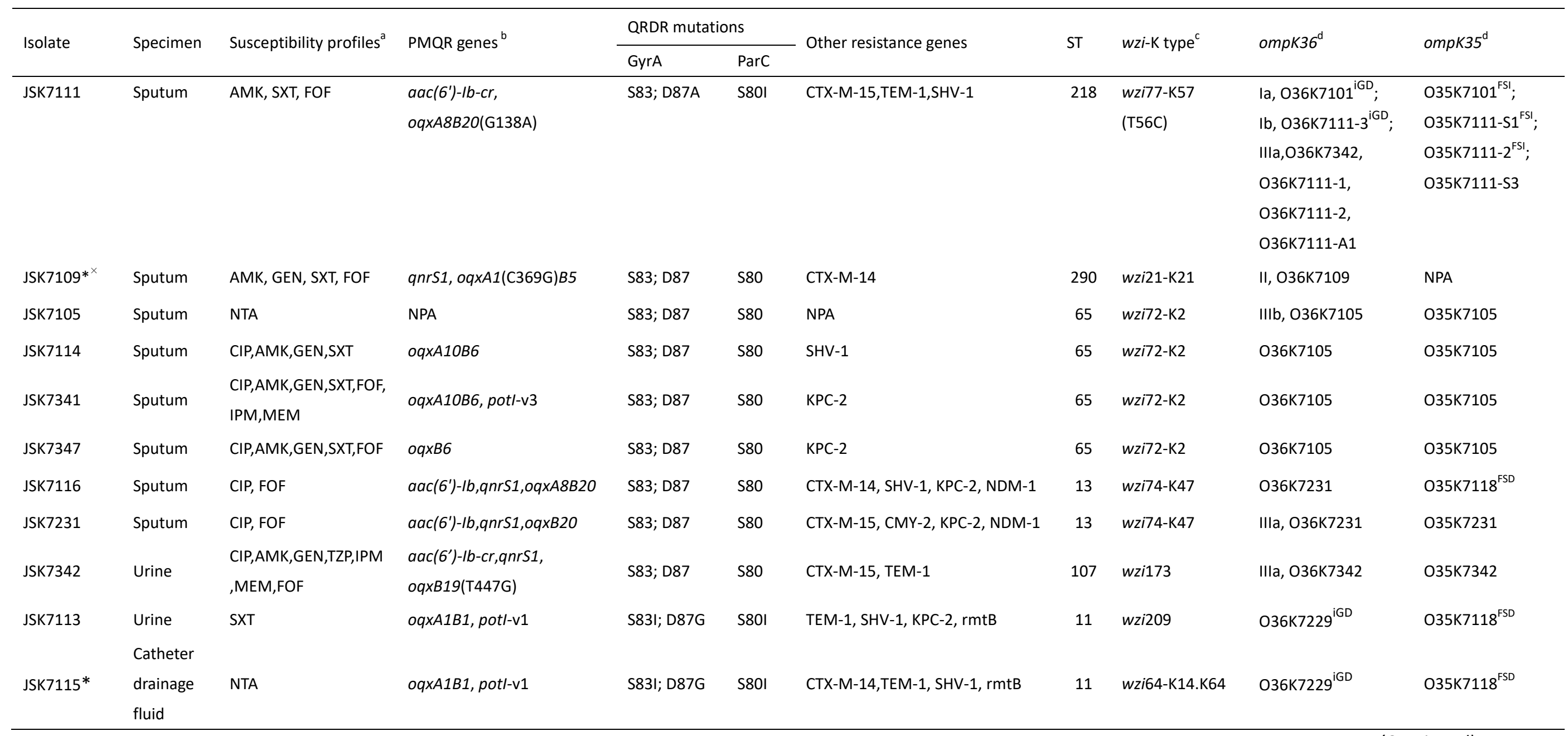


Table 1. (Continued)

\begin{tabular}{|c|c|c|c|c|c|c|c|c|c|c|}
\hline \multirow{2}{*}{ Isolate } & \multirow{2}{*}{ Specimen } & \multirow{2}{*}{ Susceptibility profiles ${ }^{a}$} & \multirow{2}{*}{ PMQR genes $^{\mathrm{b}}$} & \multicolumn{2}{|c|}{ QRDR mutations } & \multirow{2}{*}{ Other resistance genes } & \multirow{2}{*}{ ST } & \multirow{2}{*}{$w z i-K$ type $^{\mathrm{c}}$} & \multirow{2}{*}{ ompK $36^{d}$} & \multirow{2}{*}{ ompK $35^{d}$} \\
\hline & & & & GyrA & ParC & & & & & \\
\hline JSK7117* & blood & SXT & oqxA1B1 & S83I; D87G & S80I & CTX-M-14,TEM-1, KPC-2, rmtB & 11 & wzi64-K14.K64 & $036 K 7229^{\mathrm{iGD}}$ & $035 \mathrm{~K} 7118^{\mathrm{FSD}}$ \\
\hline JSK7118 & Urine & SXT & oqxA1B1, pot/-v1 & S83I; D87G & $\mathrm{S} 80 \mathrm{l}$ & $\begin{array}{l}\text { CTX-M-14,TEM-1, SHV-1, KPC-2, } \\
\text { rmtB }\end{array}$ & 11 & wzi64-K14.K64 & $036 K 7229^{\mathrm{iGD}}$ & $035 K 7118^{\text {FSD }}$ \\
\hline JSK7221 & Urine & AMK, GEN, FOF & oqxB1, potl-v1 & S83I; D87G & $\mathrm{s} 80 \mathrm{l}$ & CTX-M-15, SHV-1, KPC-2 & 11 & wzi64-K14.K64 & $036 K 7229^{\mathrm{iGD}}$ & $035 K 7118^{\mathrm{FSD}}$ \\
\hline $\operatorname{JSK} 7227^{\wedge} \times$ & Urine & SXT & oqxB1, potl-v1 & S83I; D87G & $\mathrm{s} 80 \mathrm{l}$ & CTX-M-14, KPC-2, rmtB & 11 & wzi64-K14.K64 & O36K7229 & $035 K 7118^{\mathrm{FSD}}$ \\
\hline JSK7229 & Sputum & NTA & qnrS1, potl-v1 & S83I; D87G & $\mathrm{s} 80 \mathrm{l}$ & CTX-M-14, KPC-2, rmtB & 11 & wzi64-K14.K64 & IV,036K7229 ${ }^{\mathrm{iGD}}$ & O35K7118 \\
\hline JSK7343 & Urine & FOF & $a a c\left(6^{\prime}\right)-I b-c r, q n r S 1, o q \times A 8 B 20$ & S83I; D87G & $\mathrm{s} 80 \mathrm{l}$ & CTX-M-14, KPC-2, rmtB & 11 & wzi64-K14.K64 & O36K7229 ${ }^{\mathrm{iGD}}$ & $035 \mathrm{~K} 7118^{\mathrm{FSD}}$ \\
\hline JSK7339 & Urine & $\begin{array}{l}\text { AMK,GEN,PIP,CEC,CTX,CA } \\
\text { Z,TZP,FEP,IPM,MEM,FOF }\end{array}$ & oqxA1B1, pot/-v1 & S83I; D87G & $\mathrm{s} 80 \mathrm{l}$ & SHV-1 & 11 & wzi64-K14.K64 & O36K7229 & O35K7118 ${ }^{\mathrm{FSD}}$ \\
\hline
\end{tabular}

*, severe pneumonia; ^, septicopyemia; ${ }^{\star}$, death; iGD, ins aa134-135 GD; FSD, frameshift deletion; FSI, frameshift insertion; I-IV, a-C, lineage and sublineage of ompK36 ORFs; NPA, no PCR amplification; NTA, nonsusceptible to all agents tested in this study.

a. all antimicrobial agents tested, including CIP, ciprofloxacin; AMK, amikacin; GEN, gentamicin; AMP, ampicillin; SAM, ampicillin-sulbactam; PIP, piperacillin; TZP, piperacillin-tazobactam; CFZ, cefazolin; CEC, cefaclor; CXM, cefuroxime; CTX, cefotaxime; CAZ, ceftazidime; FEP, cefepime; SXT, trimethoprim-sulfamethoxazole; FOF, fosfomycin; IMP, imipenem; MEM, meropenem.

b. pot/-v1, v2, v3 denoted three types of Putrescine ABC transporter permease (Pot/) variant (T743G $\rightarrow$ R248L). Isolates of ST11 except JSK7343 hold $100 \%$ similarity to the original oqxAB sequence of plasmid pOLA52 (EU370913), with a G148N-substitution identified in oqxB of all the other isolates.

c. Isolates of JSK7113 and JSK7342 had no associated K types.

d. Sequences of ompK36 and ompK35 have been submitted to GenBank under accession numbers MK341466-MK341478 and MK341479- MK341487, respectively. 


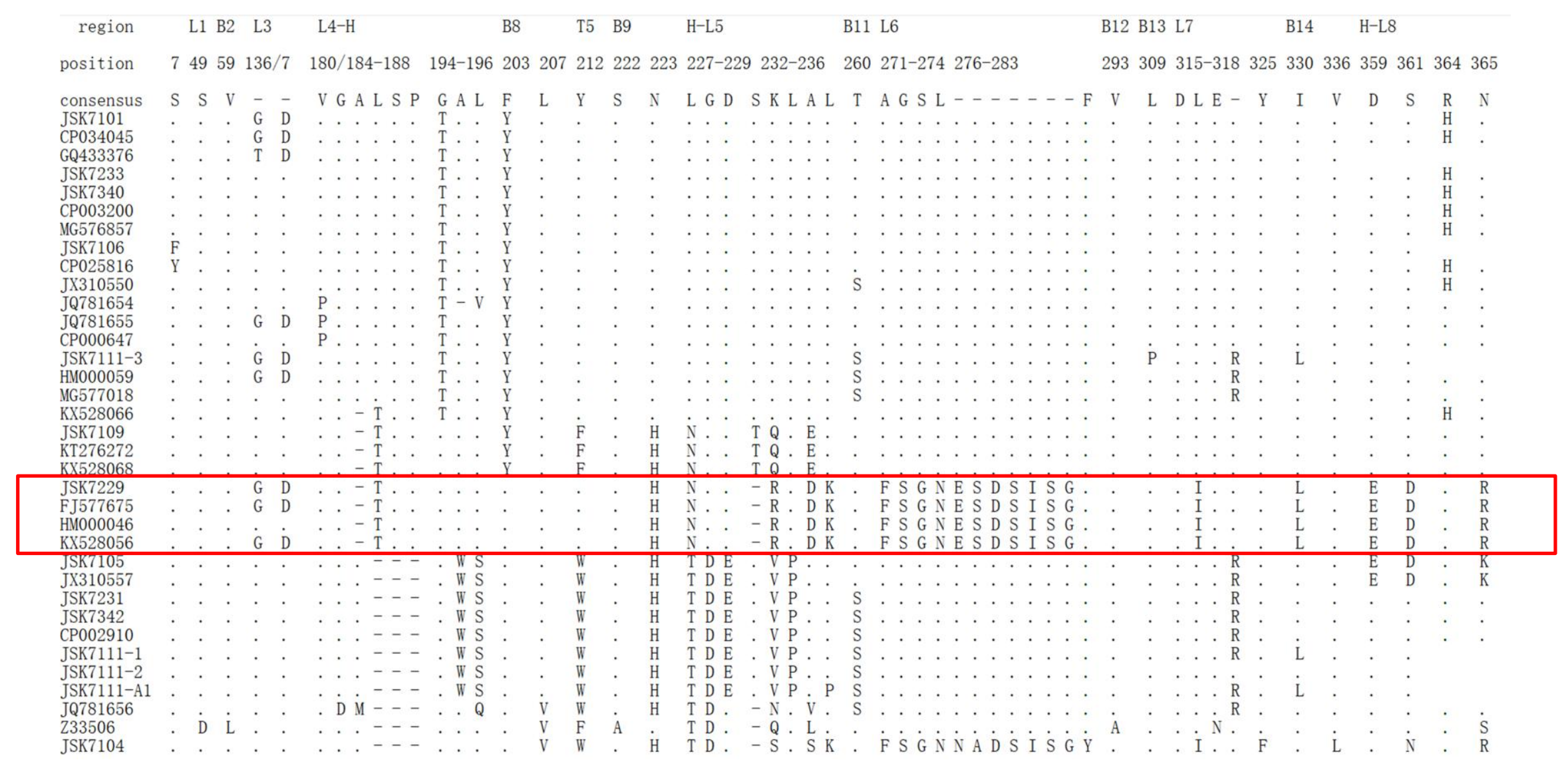

Figure 2. Comparison and alignment of Ompk36 porin regions from the representative of $K$. pneumoniae sample and reference isolates in Figure 1 . The differentiated regions included Loops (L), beta-strands $(B)$, turns $(T)$ and alpha-helix $(H)$. The consensus protein sequence was established employing the most common aa at each position in the present study. Amino acids are represented by standard single-letter codes and deletion in a site is indicated by a dash (-); those sites identical to the consensus sequence are indicated with dots. The numbers along the sequence indicated aa positions. Each sequence was designated as in the phylogenetic tree. The red box indicates the substitutions and insertions in loops of $L 3, L 4, L 5, L 6, L 7$, and $L 8$ for ST11 strains. 
Figure 3. MLST and wzi-genotyping of $34 \mathrm{~K}$. pneumonia clinical isolates. The dendrogram of wzi-alleles (447bp) was built using neighbor-joining method by MEGA program version 7.0. Bootstrap resampling (1000 replications) was used, and bootstrap values $\geq 70 \%$ were shown. Scale bar represents nt substitutions per site. Each known wzi-allele number is followed by the corresponding capsular $(K)$ type, with dots separating several $K$ types indicating cross-reactions. The three main branches (A, B, and C) are labeled. The representative wzi sequences (GenBank accession number MK341453-MK341461) of sample isolates from the present study and their identical wzi-alleles in the same cluster were shown in bold. $\bigcirc$ denotes the new wzi variants without known allele-number. The common names of isolates were followed by ward of origin, isolation-date, and MLST. ND, Neurosurgery Department; PW, Pediatric Ward. 


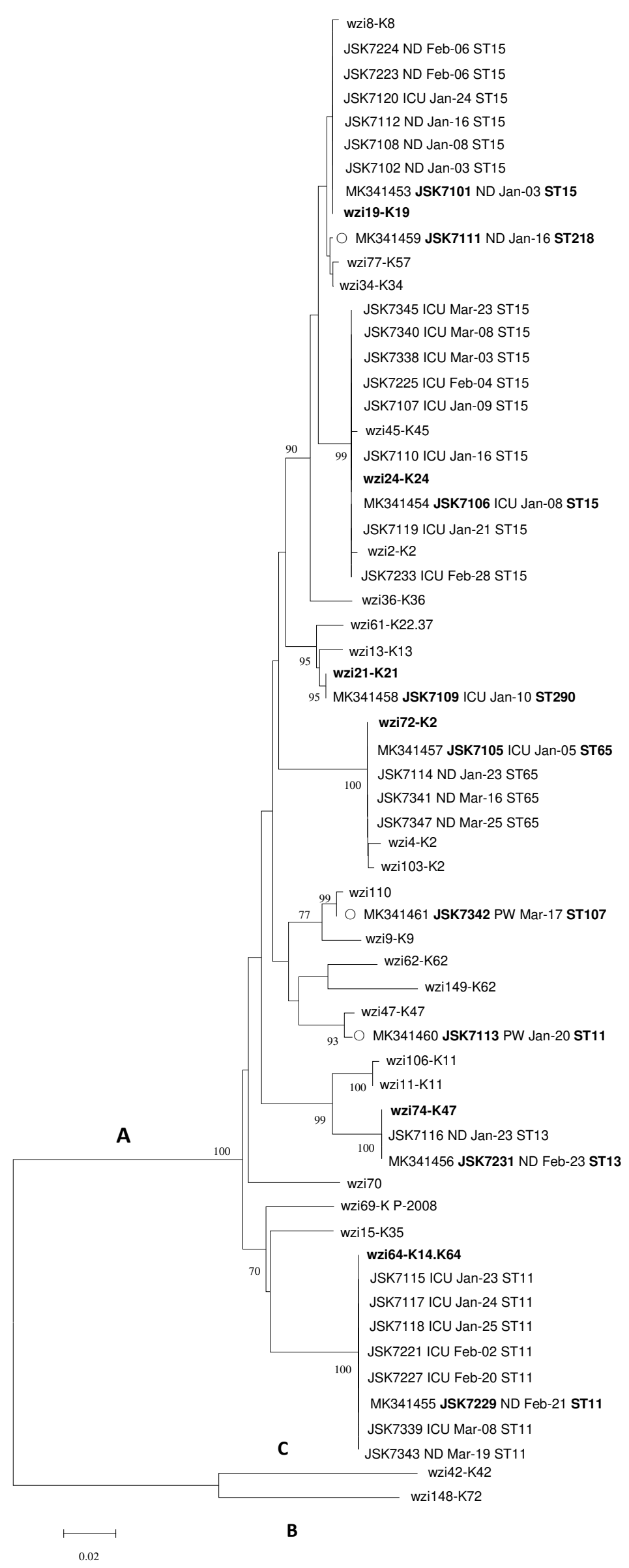

Figure 3. 


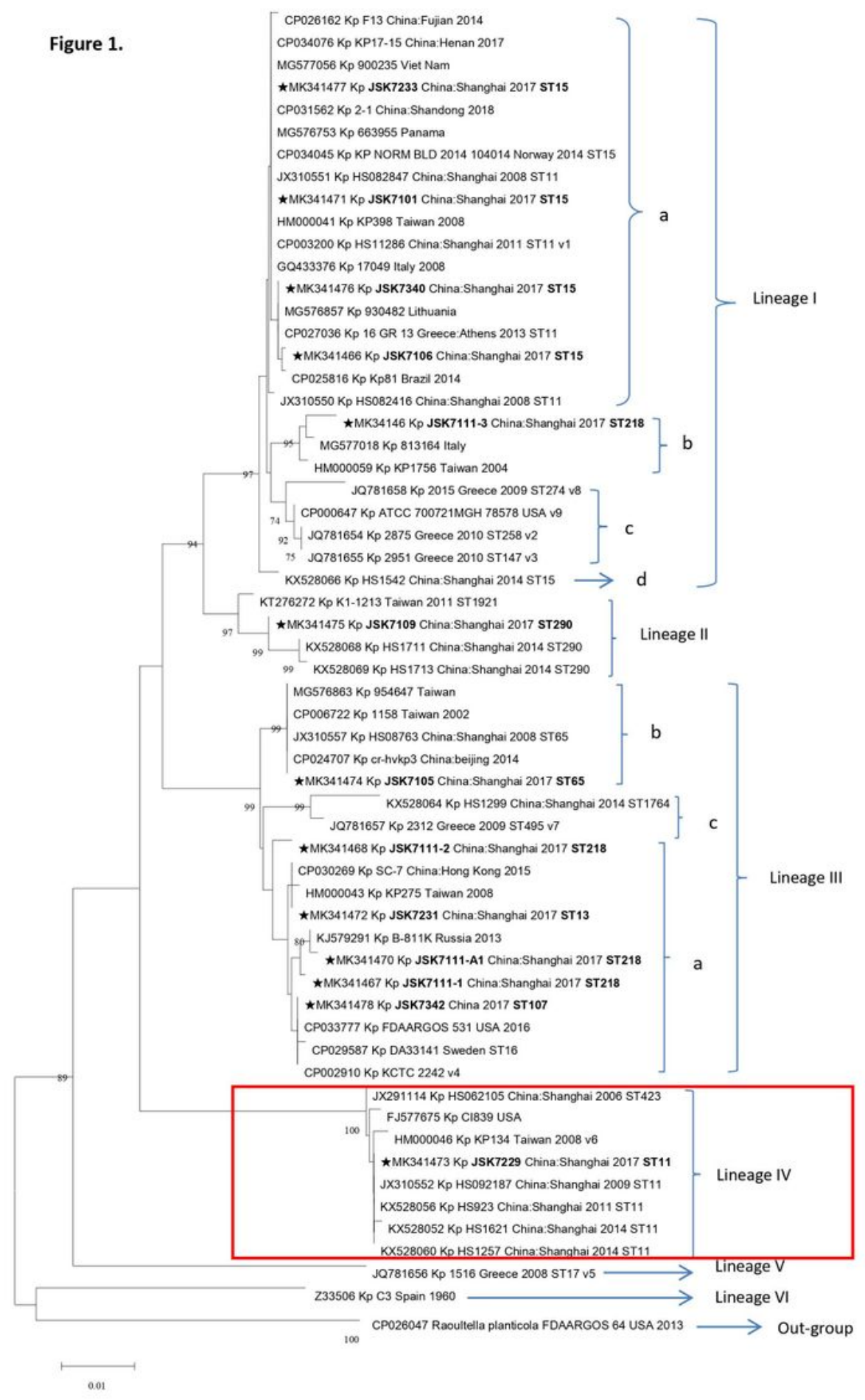

\section{Figure 1}

Please see the Manuscript PDF file for the complete figure caption 


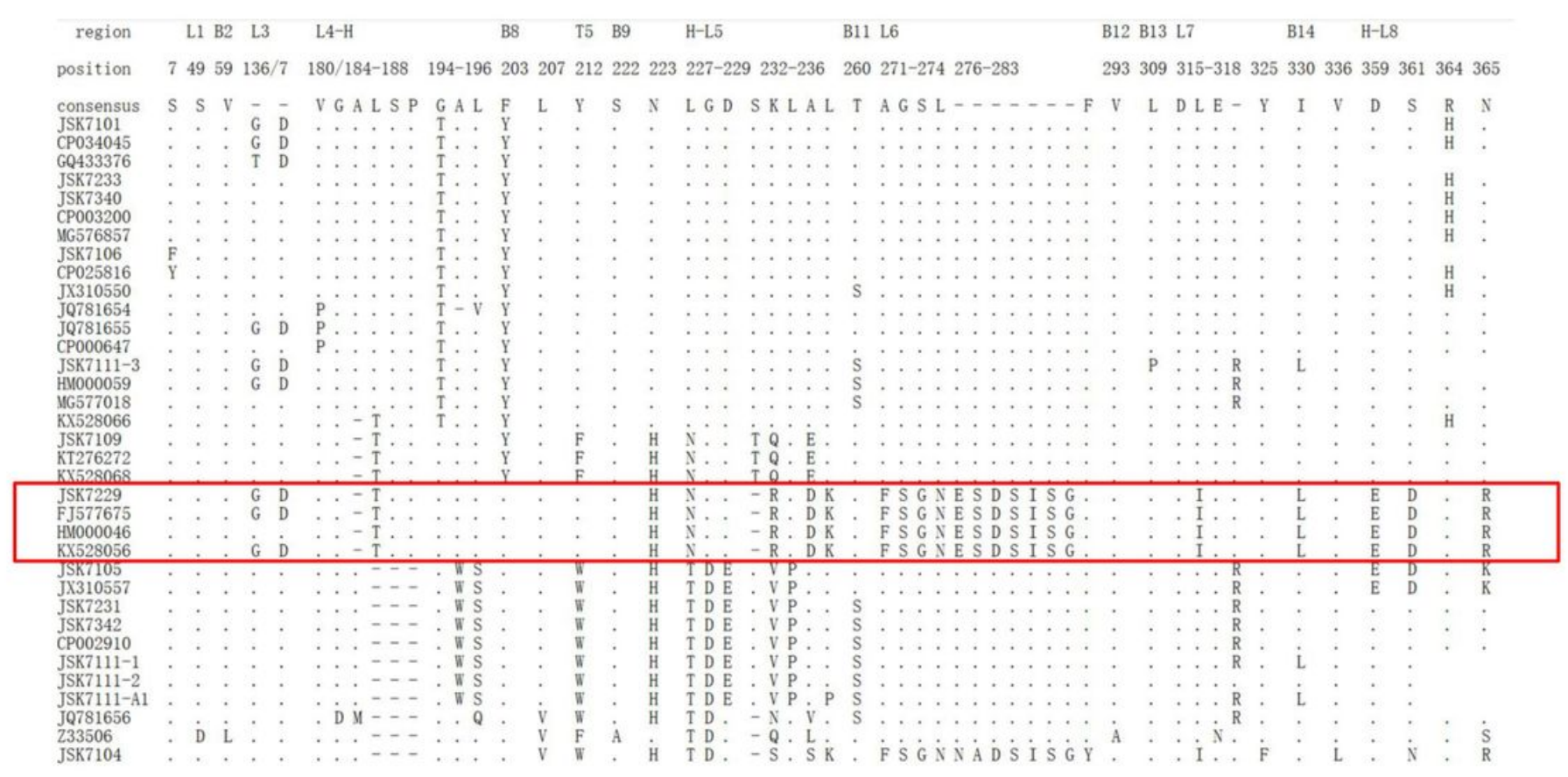

Figure 2

Please see the Manuscript PDF file for the complete figure caption 


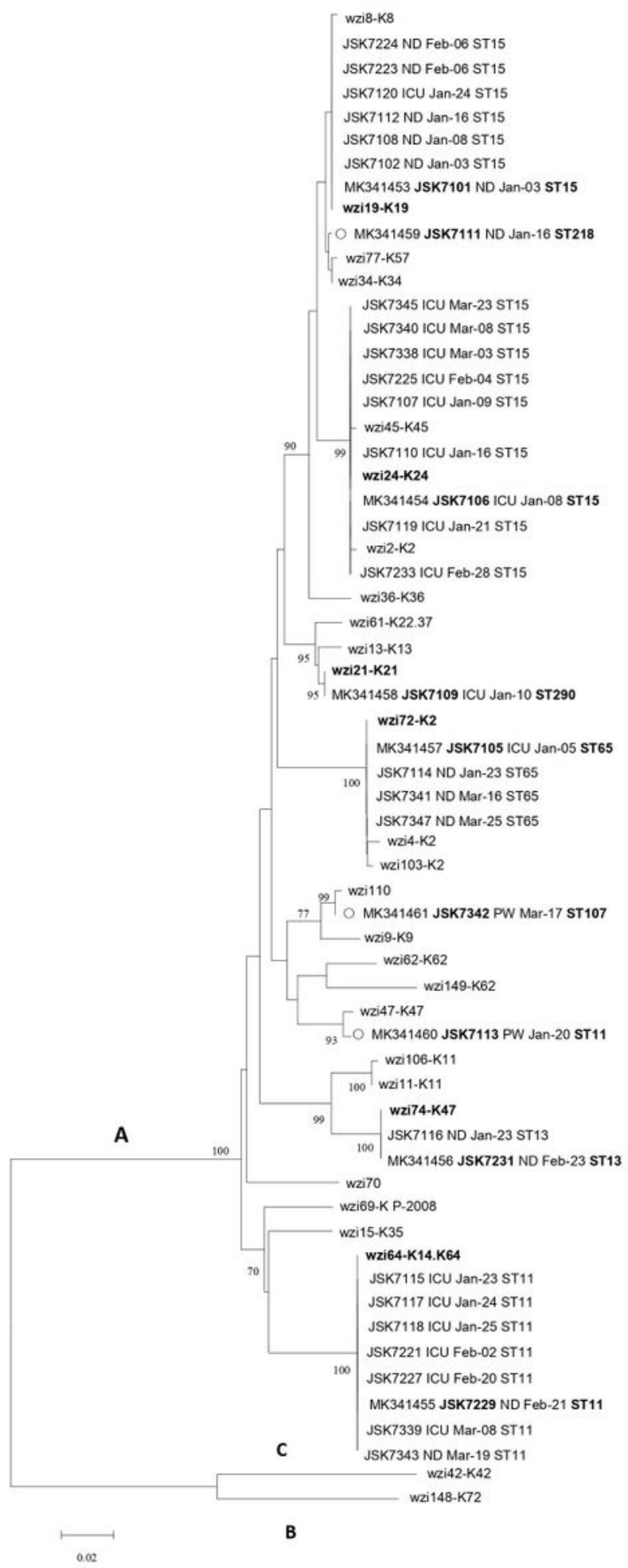

Figure 3.

\section{Figure 3}

Please see the Manuscript PDF file for the complete figure caption

\section{Supplementary Files}

This is a list of supplementary files associated with this preprint. Click to download. 
- YanWangSupplementaryMaterial.pdf 